\title{
Le sindromi mielodisplastiche: diagnosi, prognosi e terapia
}

\section{The myelodysplastic syndromes: diagnosis, prognosis and therapy}

\section{Cristina Clissa*, Carlo Finelli, Antonio de Vivo}

Istituto di Ematologia e Oncologia Clinica "Lorenzo e Ariosto Seragnòli" (Direttore: Prof. Michele Baccarani), Azienda Ospedaliero-Universitaria Policlinico "Sant'Orsola-Malpighi", Bologna

Ricevuto il 27 aprile 2010; accettato il 22 luglio 2010

disponibile online il 25 novembre 2010

\section{KEYWORDS \\ Anemia; \\ Elderly; \\ Myelodysplastic \\ syndromes; \\ Diagnosis; \\ Treatment.}

\begin{abstract}
Summary The authors conducted a systematic review of the medical literature published in the past 15 years on the myelodysplastic syndromes (MDSs). The MDSs are typically seen in the elderly, and primary and secondary forms can be distinguished. This heterogeneous group of hematologic diseases is caused by clonal disorders of pluripotent hematopoietic stem cells. The pathogenesis of the syndromes appears to be multifactorial. Genetic damage, spontaneous or induced by environmental or iatrogenic factors, leads to abnormal proliferation and apoptosis of bone marrow stem cells. The most common presentation is anemia, alone or associated with thrombocytopenia and / or neutropenia, accompanied by the related symptoms and clinical signs (asthenia, fatigue, bleeding, recurrent infections). The diagnosis involves the exclusion of other causes of cytopenia and is based on well-defined, internationally recognized criteria, which are mainly morphologic and cytogenetic. Accurate diagnosis of MDS is essential for prognostic evaluation and for estimating the risk of progression to acute myeloid leukemia (AML). The risk is rated according to the International Prognostic Scoring System (IPSS), which includes 4 levels (low, intermediate-1, intermediate-2, and high). The risk class is a major determinant of the therapeutic approach. Apart from supportive care (transfusions), the main therapeutic tools are erythropoiesis-stimulating agents (ESAs), iron-chelating agents, immunomodulatory drugs, demethylating agents and, in selected cases, allogeneic bone marrow transplantation.
\end{abstract}

(C) 2010 Elsevier Srl. All rights reserved.

\footnotetext{
* Corrispondenza: Azienda Ospedaliero-Universitaria Policlinico Sant’Orsola-Malpighi, via Massarenti 9 - 40138 Bologna.

E-mail: cristina.clissa@virgilio.it (C. Clissa).
} 


\section{Introduzione}

Le sindromi mielodisplastiche (MDS) rappresentano un gruppo eterogeneo di malattie ematologiche dovute a un disordine clonale delle cellule staminali emopoietiche pluripotenti; si caratterizzano per la presenza di eritropoiesi inefficace, una o più citopenie periferiche e aumentato rischio di evoluzione in leucemia mieloide acuta (AML) $[1,2]$.

Sono patologie tipiche dell'età adulta-anziana, la cui incidenza nella popolazione generale oscilla fra i 3 e i 12 casi ogni 100.000 abitanti/anno e aumenta in correlazione con l'età fino a 22-45 casi ogni 100.000 abitanti/anno nella popolazione ultrasettantenne [3,4]. Si distinguono forme primitive, in cui la patologia insorge de novo, e forme secondarie, in cui è possibile identificare una precedente emopatia o l'esposizione a sostanze tossiche midollari. Le forme secondarie presentano prognosi peggiore e maggiore resistenza al trattamento.

\section{Eziopatogenesi}

Nella genesi delle MDS è possibile identificare diversi fattori eziologici che contribuiscono, attraverso un processo a tappe successive, alla patogenesi della malattia.

Il danno genetico iniziale, spontaneo o indotto da cause iatrogene/ambientali, induce modificazioni dell'attività proliferativa, aumento dell'apoptosi e tendenza all'accumulo di successive mutazioni clonali responsabili della comparsa e della progressione della malattia, nonché della tendenza all'evoluzione leucemica $[4,5]$. Tra i fattori esogeni ritenuti in grado di svolgere un ruolo patogenetico determinante sono stati segnalati:

- agenti ambientali, quali l'esposizione a benzene [6], pesticidi, solventi, gas di scarico [7];

- agenti iatrogeni, quali chemioterapici citotossici (alchilanti, inibitori delle topoisomerasi, antimetaboliti e immunosoppressori), ma anche radiazioni ionizzanti e radioterapia [8];

- agenti propri dello stile di vita, come il fumo di sigaretta;

- tra le precedenti malattie ematologiche è stata invece identificata una correlazione con l'aplasia midollare e l'emoglobinuria parossistica notturna [9].

\section{Diagnosi e diagnosi differenziale}

La diagnosi di MDS richiede la presenza di alcuni criteri identificati nel 2006 da una working conference, tenutasi a Vienna, a cui hanno partecipato esperti europei e americani (US National Comprehensive Cancer Network, NCCN; International Working Group, IWG, e European Leukemia Net, ELN) [10]. Sulla base del consenso, sono stati definiti i criteri diagnostici "minimi" per formulare la diagnosi di MDS ed è stato stabilito che devono essere presenti entrambi $i$ "criteri indispensabili" in associazione con almeno uno dei "criteri decisivi".

I criteri diagnostici "indispensabili"sono:

a) citopenia prolungata ( $\geq 6$ mesi) mono o plurilineare;

b) esclusione di altre cause.
I criteri "decisivi" sono:

a) displasia morfologica mono o plurilineare (valutazione su aspirato midollare) in almeno il $10 \%$ delle cellule $(\geq 15 \%$ per i sideroblasti ad anello);

b) anomalie citogenetiche specifiche;

c) incremento delle cellule blastiche midollari (5-19\%).

Il sospetto clinico di mielodisplasia va posto ogni qualvolta ci si trovi di fronte a una citopenia, più spesso anemia, in un soggetto adulto-anziano. Qualora in anamnesi risulti un'esposizione ad agenti cancerogeni ambientali o una pregressa radio/chemioterapia, il sospetto clinico di MDS viene ulteriormente rafforzato.

Le modalità di presentazione di una MDS sono le seguenti. - Anemia isolata: definita come livello di emoglobina $(\mathrm{Hb})<13 \mathrm{~g} / \mathrm{dL}$ per il maschio e $<12 \mathrm{~g} / \mathrm{dL}$ per la femmina, secondo l'Organizzazione Mondiale della Sanità. Occorre sempre escludere cause secondarie di anemia quali disordine cronico, carenza marziale, deficit vitaminico, insufficienza renale, protesi valvolari. Nel paziente anziano l'anemia, spesso, è parte di uno scenario clinico complesso in cui più cause possono coesistere e contribuire a determinare $i$ sintomi. In corso di MDS l'anemia è più spesso normocitica o macrocitica, senza reticolocitosi periferica [11].

- Piastrinopenia isolata: è una modalità poco frequente di presentazione di una sindrome mielodisplastica all'esordio; si associa ad anemia nell' $82 \%$ dei casi. Viene definita come riduzione della conta piastrinica al di sotto di $100.000 / \mathrm{mm}^{3}$ ed è classificata in lieve, moderata o severa a seconda del valore [12]. Di fronte a una piastrinopenia isolata occorre escludere cause farmacologiche, autoimmuni (positività degli anticorpi antipiastrine o patologie autoimmuni note), una coagulazione intravasale disseminata o una microangiopatia, una malattia virale (HIV, HCV), un'epatopatia cronica con ipertensione portale e sequestro splenico.

- Neutropenia isolata: talvolta il quadro di esordio di una MDS è una neutropenia isolata che perdura nel tempo e tende a un progressivo peggioramento. All'esordio, circa due terzi dei pazienti sono neutropenici. La neutropenia viene definita come riduzione del numero assoluto dei neutrofili circolanti $\left(<1.500 / \mathrm{mm}^{3}\right)$. In relazione alla conta granulocitaria, la neutropenia può essere definita lieve, moderata e grave. Questa classificazione ha un significato clinico nel predire il rischio di infezioni batteriche, in quanto per valori $<1.000 / \mathrm{mm}^{3}$ il rischio è elevato $[13,14]$. All'osservazione delle striscio di sangue venoso periferico, inoltre, i granulociti presentano anomalie morfologiche caratterizzate da ipogranulazione del citoplasma e iposegmentazione del nucleo che a volte può essere mono o bilobato (pseudoanomalia di Pleger). L'esclusione di cause secondarie di neutropenia (autoimmuni, farmacologiche, infettive, neoplastiche) è di fondamentale importanza e richiede l'esecuzione di indagini di secondo e terzo livello quali l'agoaspirato midollare, la biopsia ossea e lo studio citogenetico.

Nel percorso diagnostico di una MDS l'esecuzione dell'agoaspirato midollare e della biopsia ossea permette di valutare la presenza di anomalie morfologiche caratteristiche della diseritropoiesi (megaloblastosi, eccesso di precursori E1-E2, frammentazioni nucleari, ponti internucleari, irregolarità cromatiniche, vacuoli citoplasmatici, siderobalsti ad anello), della disgranulopoiesi (alterazioni della lobulazione 
nucleare, assenza di granuli nel citoplasma, pseudoanomalia di Pleger) e della dismegacariocitopoiesi (presenza di micromegacariociti, piccoli megacariociti binucleati, nucleo unico non lobato o multinuclearità) [15].

Fondamentale è la quantificazione delle cellule blastiche midollari, espressa come valore percentuale, che rappresenta un parametro di riferimento per un corretto inquadramento diagnostico e prognostico.

Anche lo studio citogenetico, eseguito su sangue midollare, fornisce informazioni indispensabili per una corretta caratterizzazione della mielodisplasia e costituisce uno dei criteri "decisivi" per porre diagnosi di MDS. Sulla base del cariotipo si possono distinguere tre classi di rischio:

a) favorevole;

b) intermedio;

c) sfavorevole.

Le alterazioni più frequenti coinvolgono il cromosoma 7 , il cromosoma 5, il cromosoma 8, il braccio lungo del cromosoma $20(20 q)$ e il cromosoma $Y$ [16-18].

La presenza di una delezione interstiziale del braccio lungo del cromosoma 5 , del(5q), come unica anomalia, caratterizza un'entità clinica a sé stante definita "sindrome 5q-". Essa si distingue per alcuni aspetti peculiari: prevalenza nel sesso femminile (2:1), età di esordio media/avanzata, anemia macrocitica trasfusione-dipendente, numero di leucociti normale o ridotto e piastrinosi. La cellularità midollare può essere aumentata, normale o ridotta, con displasia eritroide, associata alla presenza di piccoli megacariociti con nucleo unico non lobato (micromegacariociti). La delezione interstiziale può essere di entità variabile e coinvolge di regola una "common deleted band" collocata nella porzione $5 q 31-32$, in cui si trovano geni coinvolti nella regolazione dell'emopoiesi. La sindrome $5 q$ - ha più spesso un decorso indolente, con sopravvivenza > 5 anni e basso rischio di evoluzione in AML. Essa, inoltre, mostra una risposta favorevole alla lenalidomide nel $70-80 \%$ dei casi $[19,20]$.

\section{Classificazione e prognosi}

Il primo sistema classificativo delle MDS fu messo a punto nel 1982 da un gruppo di ematologi europei e americani (FrenchAmerican-British, FAB) [21] sulla base di criteri esclusivamente citologici e fissava nel $30 \%$ di blasti midollari il limite tra mielodisplasia e AML.

Successivamente, nel 1999, è stata introdotta la classificazione della World Health Organization (WHO) [22,23] che ha apportato importanti modifiche, in quanto:

- è stato ridotto al $20 \%$ il valore soglia di blasti midollari per discriminare tra AML e MDS;

- è stato eliminato il gruppo AREB-t (anemia refrattaria con eccesso di blasti in trasformazione);

- si è distinta la leucemia mielomonocitica cronica (CMML) dalle altre MDS includendola in un nuovo sottogruppo (sindromi mielodisplastiche/mieloproliferative);

- è stata identificata la "sindrome del5q-" come entità a sé stante;

- è stato introdotto il sottotipo delle MDS inclassificabili (MDS-U);

- si è distinta l'anemia refrattaria (AR) dalla citopenia refrattaria con displasia multilineare (RCMD).
Recentemente tale classificazione è stata ulteriormente revisionata [24] con l'introduzione della citopenia refrattaria con displasia unilineare (RCUD), nel cui contesto si distinguono l'anemia refrattaria (AR), la trombocitopenia refrattaria (TR) e la neutropenia refrattaria (NR).

Le MDS possono essere suddivise in differenti gruppi di rischio sulla base della sopravvivenza e dell'incidenza di evoluzione in AML. In particolare, questi due parametri prognostici risultano differenti nelle MDS. Nel 1997 è stato sviluppato un sistema prognostico, denominato International Prognostic Scoring System (IPSS) [25] che, in relazione alla percentuale di cellule blastiche midollari, alle caratteristiche cariotipiche e al numero di citopenie periferiche, attribuisce uno score di rischio a ciascun paziente. È possibile così definire 4 classi principali (rischio basso, intermedio-1, intermedio-2 e alto) caratterizzate da una prognosi progressivamente peggiore e da una probabilità crescente di evoluzione in AML.

Le anomalie cariotipiche vengono distinte in 3 gruppi di differente significato prognostico: favorevole (delezione $5 q$ isolata, delezione $20 q$ e $-Y$ ), sfavorevole (anomalie del cromosoma 7 o presenza di $\geq 3$ anomalie), intermedio (qualsiasi altra anomalia).

Negli ultimi anni è stato inoltre documentato il ruolo prognostico sia della fibrosi midollare sia della trasfusionedipendenza. Una fibrosi di grado avanzato è associata a una prognosi peggiore in maniera indipendente dalla categoria WHO; anche la trasfusione-dipendenza è associata a una prognosi peggiore in termini sia di sopravvivenza sia di evoluzione leucemica, indipendentemente dal sottogruppo WHO.

Sulla base di tali osservazioni è stata formulata una nuova classificazione, il sistema "WHO classification-based Prognostic Scoring System" (WPSS) [26], che tiene conto della classificazione WHO, della citogenetica secondo l'IPSS e della richiesta trasfusionale. I pazienti vengono stratificati secondo il rischio in 5 sottogruppi: molto basso, basso, intermedio, alto, molto alto. Il WPSS permette di ottenere una stima dinamica della sopravvivenza e del rischio di trasformazione leucemica in diversi momenti della storia naturale della malattia.

\section{Approccio terapeutico}

\section{Eritropoietina}

L'eritropoietina (EPO) costituisce il più importante fattore regolatore dell'eritropoiesi, modulandone l'attività proliferativa e i processi apoptotici e rappresentando sostanzialmente un fondamentale fattore di sopravvivenza cellulare. $\grave{E}$ noto come l'ipossia tissutale conseguente a uno stato anemico rappresenti un potente stimolo alla produzione di EPO endogena. Questo fisiologico meccanismo di compenso risulta generalmente conservato in corso di MDS. Solo un terzo di tali pazienti, infatti, possiede valori di EPO endogena non adeguati rispetto al grado di anemia.

Il meccanismo d'azione dell'eritropoietina ricombinante ( $r$-HU EPO) si determinerebbe attraverso un'attività di stimolo da parte dell'ormone sull'eritropoiesi normale residua non clonale e di inibizione sui processi di apoptosi cellulare intramidollare che caratterizzano l'emopoiesi displastica. Ne deriva pertanto che la r-HU EPO sarebbe in grado di indurre 
un significativo prolungamento della sopravvivenza dei progenitori eritroidi e una riduzione dell'eritropoiesi inefficace in corso di MDS, migliorando di conseguenza i livelli di $\mathrm{Hb}$.

Uno schema di attacco con 40.000 UI di r-HU EPO alfa 2 volte/settimana per almeno 4 settimane, seguito da una somministrazione unica settimanale della stessa dose, ha indotto una percentuale di risposte globali pari al $68 \%$ in un ampio studio multicentrico italiano su pazienti mielodisplastici a basso rischio [27].

Gran parte degli studi clinici finora condotti ha evidenziato come i pazienti non trasfusione-dipendenti e con livelli di EPO endogena non elevati (200-500 mUl/mL) abbiano le maggiori possibilità di rispondere a un trattamento con $\mathrm{r}-\mathrm{HU}$ EPO.

\section{Fattori di crescita per il trattamento delle altre citopenie}

G-CSF e GM-CSF (Granulocyte-Colony Stimulating Factor e Granulocyte-Macrophage Colony Stimulating Factor) sono stati usati in profilassi, a dosi e con schemi variabili, in pazienti con MDS allo scopo di migliorare la neutropenia e ridurre il rischio infettivo. Nel 76-90\% dei pazienti trattati è stato possibile ottenere un incremento significativo dei leucociti e, in particolare, del numero di granulociti [28,29]. Non è stato tuttavia dimostrato un impatto significativo sulla percentuale e sulla gravità degli eventi infettivi e sulla sopravvivenza globale. In base alle esperienze pubblicate, l'uso dei fattori di crescita mieloidi, in particolare del G-CSF, può essere preso in considerazione esclusivamente in pazienti con grave neutropenia febbrile resistente alla terapia antinfettiva (antibatterica e/o antifungina).

\section{Supporto trasfusionale}

La trasfusione di eritrociti e piastrine costituisce il cardine della terapia di supporto, insieme alla terapia ferrochelante e alla profilassi e trattamento delle complicanze infettive.

L'anemia rappresenta il problema clinico più comune nelle MDS: la si riscontra nel $50 \%$ circa dei pazienti al momento della diagnosi e comunque in quasi tutti durante il decorso successivo della malattia, per cui l'80\% dei pazienti prima o poi necessita di trasfusioni di eritrociti.

In assenza di comorbilità importanti a carico dell'apparato cardiovascolare o respiratorio, di solito l'anemia è poco sintomatica per livelli di $\mathrm{Hb}$ compresi tra 11 e $10 \mathrm{~g} / \mathrm{dL}$, mentre fra 9 e $7,5 \mathrm{~g} / \mathrm{dL}$ cominciano a comparire astenia importante e dispnea da sforzo; al di sotto di 7,5 g/dL astenia e dispnea sono presenti anche a riposo. Per quanto riguarda il target ottimale di $\mathrm{Hb}$ da raggiungere con la terapia trasfusionale, recenti acquisizioni pongono in discussione la linea di condotta seguita fino a oggi dalla maggior parte dei Centri, e cioè di mantenere un livello di Hb compreso fra 8 e $9 \mathrm{~g} / \mathrm{dL}$ nella maggior parte dei pazienti. Numerosi studi hanno evidenziato che la qualità di vita (QoL) dei pazienti mielodisplastici anziani è spesso insoddisfacente, a motivo dell'anemia cronica che si associa a un insieme di sintomi fisici e psichici denominati "fatigue" ed è strettamente correlata con il livello di $\mathrm{Hb}[30,31]$; incrementando la concentrazione emoglobinica a seguito di un trattamento con eritropoietina ricombinante, la QoL mostra un miglioramento e il massimo beneficio in termini di QoL si ottiene se l'Hb raggiunge valori di $12 \mathrm{~g} / \mathrm{dL}$.

La trasfusione di piastrine ha lo scopo di prevenire e trattare le complicanze emorragiche causate dalla piastrinopenia. Attualmente l'indicazione alla trasfusione piastrinica in caso di emorragia in atto è universalmente accettata, mentre l'utilità del supporto piastrinico a scopo profilattico è controversa e non supportata da sufficienti evidenze.

\section{Ferrochelazione}

È noto che il sovraccarico marziale correla con un prognosi peggiore nei pazienti trasfusione-dipendenti. La terapia ferrochelante costituisce il principale strumento terapeutico per prevenire e correggere il sovraccarico marziale, in quanto l'organismo umano non riesce da solo a eliminare il ferro in eccesso. L'entità del sovraccarico marziale può essere valutata con metodi diretti e indiretti:

- valutazione della ferritina sierica;

- misurazione della quota di ferro non legato alla transferrina (Non Transferrin Bound Iron, NTBI), metodica a tutt'oggi sperimentale;

- valutazione funzionale dei vari organi (cuore, fegato, sistema endocrino);

- biomagnetometria o Superconducting Quantum Interference Device (SQUID);

- risonanza magnetica (RMN) del fegato e del cuore.

La determinazione della ferritina sierica (espressa in ng/ $\mathrm{mL}$ ) costituisce il metodo più comunemente impiegato per valutare l'entità dei depositi marziali dell'organismo; i valori normali sono compresi fra 20 e $230 \mathrm{ng} / \mathrm{mL}$ nei soggetti sani. A ogni $\mathrm{ng} / \mathrm{mL}$ di ferritina corrispondono $8 \mathrm{mg}$ di ferro di deposito [32]. Il monitoraggio della ferritinemia a intervalli regolari (per esempio, ogni 3 mesi) può fornire indicazioni utili nei pazienti sottoposti a terapia chelante. Il mantenimento di una ferritinemia $<1.000 \mathrm{ng} / \mathrm{mL}$ costituisce un obiettivo comunemente accettato della terapia ferrochelante nel paziente trasfusione-dipendente e livelli $>2.500 \mathrm{ng} / \mathrm{mL}$ sono associati a un aumentato rischio di complicanze cardiache [33].

Attualmente i farmaci ferrochelanti disponibili sono tre: deferoxamina, deferiprone, deferasirox [34].

- La deferoxamina (DFO), scoperta nel 1960, all'inizio veniva somministrata per via intramuscolare, successivamente in infusione continua di 24 ore per via endovenosa. Gli effetti collaterali più importanti osservati in corso di trattamento con DFO sono costituiti da: reazioni locali nel sito d'infusione, ipersensibilità al farmaco, tossicità oftalmica, ototossicità, alterazioni dell'accrescimento osseo, aumentato rischio di infezione da Yersinia. Le dosi terapeutiche standard sono di $30-50 \mathrm{mg} / \mathrm{kg} / \mathrm{die}$ in $8-10$ ore di infusione sottocutanea 5 giorni/settimana. In casi particolari (per esempio, pazienti con emosiderosi molto grave e/o con complicanze cardiache) la dose può essere aumentata fino a $50-60 \mathrm{mg} / \mathrm{kg} / \mathrm{die}$ in infusione continua per 24 ore mediante catetere venoso centrale.

- Il primo chelante orale idoneo all'uso terapeutico nell'uomo è stato l'1,2-dimetil-3-idrossipiridina-4-1, denominato anche L1 o deferiprone. In considerazione delle sue proprietà farmacocinetiche, è necessaria una somministrazione frazionata ( 3 volte al giorno). Tuttavia tale farmaco 
non può essere utilizzato in pazienti affetti da MDS per il rischio di agranulocitosi.

- Negli ultimi anni la ricerca farmacologica ha continuato a testare nuovi farmaci chelanti potenzialmente attivi per via orale. Fra questi l'ICL670, o deferasirox, ha già ricevuto l'approvazione da parte delle autorità regolatorie negli Stati Uniti (2005), in Europa (2006) e in Italia (2007). È un chelante del ferro potente e selettivo, escreto principalmente con la bile, in grado di provocare un incremento dell'eliminazione del ferro per via fecale superiore di 4-5 volte rispetto al DFO. La sua attività ferrochelante è risultata superiore di 5 volte rispetto alla DFO e di 10 volte rispetto a L1. Le caratteristiche farmacocinetiche dell'ICL670 ne consentono la somministrazione in un'unica dose giornaliera. Il farmaco, disciolto in acqua, va assunto a stomaco vuoto almeno mezz'ora prima del pasto. Quanto alla tossicità dell'ICL670 [35], i dati globali di tutti gli studi finora condotti hanno confermato la soddisfacente tollerabilità mostrata dai primi studi. Gli effetti collaterali più frequenti sono di tipo gastrointestinale (15\% dei pazienti: dolori addominali, nausea, vomito, diarrea, stipsi), rush cutaneo (11\% dei casi). Il problema della tossicità renale è controverso: circa un terzo dei pazienti (38\%) mostra un incremento moderato della creatininemia ( $\geq 33 \%$ rispetto al valore basale). Tale evento è dose-dipendente: si manifesta più frequentemente alle posologie più elevate (20$30 \mathrm{mg} / \mathrm{kg} / \mathrm{die}$ ). L'aumento della creatininemia è comunque generalmente di moderata entità, spesso transitorio e per lo più si arresta o regredisce a seguito della riduzione della posologia. È necessaria tuttavia una particolare cautela per quanto riguarda la funzionalità renale, che deve essere controllata con cadenza almeno mensile.

In sintesi, secondo una recente revisione delle linee guida della Società Italiana di Ematologia (SIE) [36], la terapia ferrochelante è raccomandata:

a) nei pazienti con MDS a rischio basso/intermedio-1 che ricevono regolarmente supporto trasfusionale eritrocitario, nei quali va iniziata dopo 20 unità di globuli rossi concentrati;

b) nei pazienti trasfusione-dipendenti con MDS a rischio alto/ intermedio-2 se responsivi a terapie in grado di modificare la loro aspettativa di vita o se possibili candidati a trapianto allogenico di midollo osseo.

I pazienti per i quali la terapia ferrochelante orale è controindicata o non tollerata dovrebbero essere trattati con deferoxamina per via sottocutanea per 8-10 ore/die.

\section{Lenalidomide}

È un farmaco immunomodulante, con potente attività di inibizione del TNF-alfa (Tumor Necrosis Factor-alpha), VEGF (Vascular Endotelium Growth Factor), IL-6 (interleuchina-6) e dell'angiogenesi, in grado di stimolare la proliferazione T cellulare, l'attività citotossica delle cellule NK e la produzione di IL-2 e interferone-gamma. La sua efficacia è stata dimostrata, nell'ambito delle MDS, da uno studio preliminare di fase I/II, in cui la lenalidomide è stata somministrata al dosaggio di $10 \mathrm{mg} /$ die in 10 su 12 pazienti mielodisplastici con alterazione citogenetica $\operatorname{del}(5 q)$ rendendoli trasfusioneindipendenti e inducendo risposte citogenetiche complete e parziali [37].
La Food and Drug Administration (FDA) statunitense ha approvato nel dicembre 2005 l'uso della lenalidomide per i pazienti affetti da MDS trasfusione-dipendenti, a rischio IPSS basso o intermedio-1, con delezione $5 q$ (5q13-33) isolata o associata ad altre anomalie del cariotipo [38]. Tale anomalia è presente nel $15-18 \%$ circa di tutti i pazienti con MDS primitiva e fino al $50 \%$ di quelli con MDS secondaria o correlata a trattamenti chemioterapici [39].

Poiché la lenalidomide viene prevalentemente eliminata per via renale, sono necessari un attento monitoraggio della funzionalità d'organo ed eventuali aggiustamenti del dosaggio in pazienti con valori di creatinina elevati e/o ridotta clearance. Non è a tutt'oggi noto con quali modalità la lenalidomide esplichi la sua azione nei soggetti mielodisplastici e, in particolare, in quelli con delezione $5 q-$. Al di là di possibili attività sul microambiente midollare, è evidente che il farmaco esercita un importante effetto diretto sul clone neoplastico. Sono stati identificati alcuni geni oncosoppressori o dotati di attività proteinchinasica, modulante i processi apoptotici o codificanti per proteine ribosomiali, potenzialmente coinvolti nella patogenesi della sindrome del $5 q$ $[40,41]$. La loro rimodulazione indotta dalla lenalidomide potrebbe essere alla base della particolare sensibilità al trattamento di questi pazienti, fornendo in tal modo un nuovo possibile esempio di "target-therapy" molecolare.

Secondo la recente revisione delle linee guida della SIE [36], il trattamento con lenalidomide, alla dose iniziale di 5$10 \mathrm{mg} /$ die per via orale 21 giorni/mese per almeno 4 cicli, è indicato per pazienti con MDS a rischio basso/intermedio-1, trasfusione-dipendenti e portatori di delezione $5 q$ (isolata 0 in combinazione con altre anomalie citogenetiche).

\section{Azacitidina e farmaci demetilanti}

I farmaci inibitori delle DNA-metiltransferasi sono agenti in grado di bloccare i processi di ipermetilazione responsabili del silenziamento genico; è stato dimostrato che un'aumentata metilazione del DNA a carico di geni oncosoppressori quali p15ink4b, E-cadherin, HIC, rappresenta uno dei meccanismi molecolari responsabili della genesi e progressione dei tumori, ematologici e non, e sembra essere ampiamente coinvolta nella patogenesi delle mielodisplasie $[42,43]$. L'azacitidina (AZA) è un farmaco noto da oltre 40 anni, inizialmente utilizzato a dosi elevate per i suoi effetti citotossici nella terapia delle leucemie acute, ma gravato da maggiori tossicità rispetto alla citarabina. Recentemente tale molecola è stata invece indagata nell'ambito delle MDS per la sua azione demetilante; l'AZA, infatti, sembra interferire con l'attività dell'enzima DNA-metiltransferasi bloccando definitivamente i processi di ipermetilazione.

Il suo impiego clinico è stato approvato nel 2004 dall'FDA [44] per il trattamento delle MDS a seguito di alcune importanti pubblicazioni [45-47], le principali delle quali a opera del Cancer and Leukemia Group B (CALGB). In particolare, l'efficacia dell'AZA è stata comprovata nello studio randomizzato di fase III CALGB 9221 [48], in cui 191 pazienti anziani affetti da MDS ad alto rischio sono stati randomizzati a ricevere AZA sottocute $75 \mathrm{mg} / \mathrm{m}^{2} /$ die per 7 giorni ogni 4 settimane oppure, come braccio di controllo, la sola terapia di supporto, escluso l'uso di fattori di crescita emopoietici. Mentre la mortalità correlata alla terapia è stata molto 
bassa, la risposta globale ha raggiunto il 60\% e nel braccio di controllo il $5 \%$. La durata media di risposta è stata di 15 mesi. Il $15 \%$ dei pazienti in terapia con AZA è evoluto in AML, mentre i pazienti in terapia di supporto sono evoluti nel $38 \%$ dei casi. L'impatto della terapia con AZA sulla QoL dei pazienti con mielodisplasia è stato valutato in questo studio CALGB mediante un questionario messo a punto dalla European Organisation for Research and Treatment of Cancer (EORTC). I risultati hanno indicato una significativa diminuzione di astenia, dispnea e stress psicologico, con migliorata funzione fisica nei pazienti riceventi AZA rispetto a quelli del braccio di controllo.

Lo studio più importante, che ha definitivamente dimostrato l'impatto positivo del trattamento con AZA sulla sopravvivenza, è stato quello di Fenaux et al. [49] in cui sono stati arruolati 358 pazienti, classificati secondo il sistema FAB e a rischio IPSS alto o intermedio-2. Il trattamento con AZA alla dose standard di $75 \mathrm{mg} / \mathrm{m}^{2} /$ die per 7 giorni ogni 28 è stato confrontato con la terapia convenzionale (sola terapia di supporto, citosina arabinoside a basse dosi oppure chemioterapia antileucemica convenzionale, antracicline + citarabina). I risultati ottenuti hanno documentato una maggiore sopravvivenza nel braccio trattato con AZA rispetto al braccio di controllo (24,5 vs 15 mesi), una maggiore efficacia in termini di percentuale di risposta e un ritardo della progressione in AML.

Recentemente sono stati condotti studi per confrontare l'efficacia di AZA impiegata secondo diversi regimi terapeutici che permettessero di evitare la somministrazione del farmaco durante il finesettimana fornendo un significativo vantaggio in termini di farmacoeconomia e compliance da parte dei pazienti. Il più vasto studio multicentrico randomizzato di fase Il è stato condotto negli Stati Uniti [50]; sono stati arruolati 151 pazienti con mielodisplasia prevalentemente a rischio basso e trattati secondo tre differenti schemi terapeutici:

- AZA 5-2-2: AZA $75 \mathrm{mg} / \mathrm{m}^{2} /$ die per 7 giorni, con 2 giorni di intervallo nel finesettimana;

- AZA 5-2-5: AZA $50 \mathrm{mg} / \mathrm{m}^{2} /$ die per 10 giorni, con 2 giorni di intervallo nel finesettimana;

- AZA 5: AZA $75 \mathrm{mg} / \mathrm{m}^{2} /$ die per 5 giorni consecutivi.

Confrontando sia la percentuale di risposte ottenute sia la tossicità i risultati non sono apparsi significativamente diversi nei tre diversi gruppi di pazienti trattati.

Nel dicembre 2008 l'impiego di AZA è stato approvato anche dalla Commissione Europea in pazienti adulti con MDS a rischio alto e intermedio-2 secondo I'IPSS, leucemia mielomonocitica cronica (LMMC) con il 10-29\% di blasti midollari e AML con $20-30 \%$ di blasti e displasia multilineare.

Secondo le linee guida della SIE [36] la terapia demetilante è indicata:

- in pazienti con MDS a rischio alto/intermedio-2 non eleggibili al trapianto allogenico di midollo osseo o quando non sia immediatamente disponibile un donatore;

- in pazienti con MDS a rischio basso/intermedio-1, in assenza di delezione $5 q$ e in presenza di almeno una delle seguenti condizioni: non indicazione alla terapia con EPO, citopenia severa sintomatica, oltre il $5 \%$ di blasti midollari, caratteristiche citogenetiche sfavorevoli. Inoltre, i pazienti con IPSS basso/intermedio-1 sono candidati a terapia con agenti ipometilanti in caso di dimostrata resistenza al trattamento immunosoppressivo, immunomodulante o con EPO.
Negli ultimi anni, studi condotti presso il Dipartimento di Anatomia Umana, in collaborazione con l'Istituto di Ematologia dell'Università di Bologna, hanno messo in evidenza una correlazione esistente tra l'espressione del gene di una fosfolipasi, la PI-PLC-beta1, coinvolta nel controllo della proliferazione e differenziazione cellulare, e la risposta al trattamento demetilante con AZA in pazienti affetti da MDS, suggerendo così l'ipotesi di un marcatore molecolare utile per il monitoraggio della risposta alla terapia [51-53].

\section{Trapianto allogenico di midollo osseo}

Le MDS, in quanto patologie tipiche dell'età adulta-anziana, costituiscono raramente indicazione al trapianto allogenico di cellule staminali emopoietiche. In questa fascia d'età, infatti, la mortalità e la morbilità trapianto-correlate sono molto alte, la malattia tende spesso ad avere un decorso lento e nuove acquisizioni terapeutiche hanno prolungato e migliorato l'aspettativa di vita e la QoL.

In relazione all'età, il trapianto allogenico è indicato in pazienti meno anziani ( $<55$ anni) con mielodisplasia a rischio alto (intermedio-2 o alto, secondo l'IPSS), che abbiano un donatore HLA-compatibile familiare o volontario (MUD). In mancanza di un donatore compatibile e di un MUD in tempi brevi, è possibile eseguire il trapianto da donatore aploidentico o da cordone ombelicale [54].

Per pazienti di età compresa tra 55 e 65 anni oppure per pazienti con importanti comorbilità, l'opzione del trapianto allogenico è più rischiosa, ma comunque possibile adottando regimi di condizionamento a intensità ridotta, dotati di minore tossicità extraematologica.

Al contrario, nel caso di pazienti di età $<55$ anni con MDS a rischio basso o intermedio-1, anche in presenza di un donatore HLA-identico, è indicato aspettare, monitorare il paziente con uno stretto follow-up clinico, ematologico e citogenetico e procedere al trapianto solo in caso di progressione della malattia o non risposta alla terapia, con necessità di elevato supporto trasfusionale e importante compromissione della QoL [55].

Secondo la recente revisione delle linee guida della SIE [36], nei pazienti di età $<65$ anni dovrebbe essere valutata l'eleggibilità al trapianto allogenico e i migliori candidati sono:

a) i pazienti con rischio IPSS alto o intermedio-2:

b) anche i pazienti a rischio IPSS più basso, ma con caratteristiche di maggiore aggressività (per esempio, importante fabbisogno trasfusionale, piastrinopenia e/o neutropenia severa, citogenetica sfavorevole, blastosi midollare > $5 \%)$.

\section{Conflitto di interesse}

Gli autori dichiarano di essere esenti da conflitto di interessi.

\section{Bibliografia}

[1] Heaney ML, Golde DW. Myelodysplasia. N Engl J Med 1999; 340(21):1649-60.

[2] List AF, Doll DC. The myelodysplastic syndromes. In: Wintrobe's Clinical Hematology $10^{\text {th }}$ Ed., Philadelphia, PA: Lippincott Williams \& Wilkins; 1999. p. 2320. 
[3] Williamson PJ, Kruger AR, Reynolds PJ, Hamblin TJ, Oscier DG. Establishing the incidence of myelodysplastic syndrome. $\mathrm{Br} \mathrm{J}$ Haematol 1994;87(4):743-5.

[4] Aul C, Bowen DT, Yoshida Y. Pathogenesis, etiology and epidemiology of myelodysplastic syndromes. Haematologica 1998; 83(1):71-86.

[5] Levis A, Salvi F. Eziologia ed epidemiologia. In: Aloe Spiriti MA, Cortelezzi A, Finelli C, et al., editors. Sindromi mielodisplastiche. Dalla teoria alla pratica clinica. Milano: Elsevier Masson; 2008. p. 17.

[6] Morgan GJ, Alvares CL. Benzene and the hemopoietic stem cell. Chem Biol Interact 2005;153-154:217-22.

[7] Descatha A, Jenabian A, Conso F, Ameille J. Occupational exposures and haematological malignancies: overview on human recent data. Cancer Causes Control 2005;16(8): 939-53.

[8] Cardis E, Vrijheid M, Blettner M, Gilbert E, Hakama M, Hill C, et al. Risk of cancer after low doses of ionising radiation: retrospective cohort study in 15 countries. BMJ 2005; 331(7508): 77.

[9] Socié G, Mary JY, de Gramont A, Rio B, Leporrier M, Rose C, et al. Paroxysmal nocturnal haemoglobinuria: long-term follow-up and prognostic factors. French Society of Haematology. Lancet 1996;348(9027):573-7.

[10] Valent P, Horny HP, Bennett JM, Fonatsch C, Germing U, Greenberg P, et al. Definitions and standards in the diagnosis and treatment of the myelodysplastic syndromes: Consensus statements and report from a working conference. Leuk Res 2007;31(6):727-36.

[11] Ghio R, Levis A, Santini V. Il sospetto clinico. In: Aloe Spiriti MA, Cortelezzi A, Finelli C, et al, editors. Sindromi mielodisplastiche. Dalla teoria alla pratica clinica. Milano: Elsevier Masson; 2008. p. 29.

[12] Kantarjian H, Giles F, List A, Lyons R, Sekeres MA, Pierce S, et al. The incidence and impact of thrombocytopenia in myelodysplastic syndromes. Cancer 2007;109(9):1705-14.

[13] Pomeroy C, Oken MM, Rydell RE, Filice GA. Infection in the myelodysplastic syndromes. Am J Med 1991;90(3):338-44.

[14] Neth OW, Bajaj-Elliott M, Turner MW, Klein NJ. Susceptibility to infection in patients with neutropenia: the role of the innate immune system. Br J Haematol 2005;129(6):713-22.

[15] Liso V. Diagnostica morfologica. In: Aloe Spiriti MA, Cortelezzi A, Finelli C, et al, editors. Sindromi mielodisplastiche. Dalla teoria alla pratica clinica. Milano: Elsevier Masson; 2008. p. 47.

[16] Vallespí T, Imbert M, Mecucci C, Preudhomme C, Fenaux P. Diagnosis, classification, and cytogenetics of myelodysplastic syndromes. Haematologica 1998;83(3):258-75.

[17] Olney HJ, Le Beau MM. The cytogenetics of myelodysplastic syndromes. Best Pract Res Clin Haematol 2001;14(3):479-95.

[18] Bernasconi P, Klersy C, Boni M, Cavigliano PM, Calatroni S, Giardini I, et al. Incidence and prognostic significance of karyotype abnormalities in de novo primary myelodysplastic syndromes: a study on 331 patients from a single institution. Leukemia 2005;19(8):1424-31.

[19] Giagounidis AA, Germing U, Haase S, Hildebrandt B, Schlegelberger B, Schoch C, et al. Clinical, morphological, cytogenetic, and prognostic features of patients with myelodysplastic syndromes and del $(5 q)$ including band $q 31$. Leukemia 2004;18(1):113-9.

[20] Lewis S, Oscier D, Boultwood J, Ross F, Fitchett M, Rack K, et al. Hematological features of patients with myelodysplastic syndromes associated with a chromosome $5 \mathrm{q}$ deletion. Am J Hematol 1995;49(3):194-200.

[21] Bennett JM, Catovsky D, Daniel MT, Flandrin G, Galton DA, Gralnick HR, et al. Proposals for the classification of the myelodysplastic syndromes. Br J Haematol 1982;51(2):189-99.

[22] Harris NL, Jaffe ES, Diebold J, Flandrin G, Muller-Hermelink HK, Vardiman J, et al. World Health Organization classification of neoplastic diseases of the hematopoietic and lymphoid tissues: report of the Clinical Advisory Committee meeting-Airlie House, Virginia, November 1997. J Clin Oncol 1999;17(12):3835-49.

[23] Vardiman JW, Harris NL, Brunning RD. The World Health Organization (WHO) classification of the myeloid neoplasms. Blood 2002;100(7):2292-302.

[24] Vardiman JW, Thiele J, Arber DA, Brunning RD, Borowitz MJ, Porwit A, et al. The 2008 revision of the World Health Organization (WHO) classification of myeloid neoplasms and acute leukemia: rationale and important changes. Blood 2009;114(5): 937-51.

[25] Greenberg P, Cox C, LeBeau MM, Fenaux P, Morel P, Sanz G, et al. International scoring system for evaluating prognosis in myelodysplastic syndromes. Blood 1997;89(6):2079-88.

[26] Malcovati L, Germing U, Kuendgen A, Della Porta MG, Pascutto C, Invernizzi R, et al. Time-dependent prognostic scoring system for predicting survival and leukemic evolution in myelodysplastic syndromes. J Clin Oncol 2007;25(23):3503-10.

[27] Aloe Spiriti MA, Petti MC, Latagliata R, et al., the Italian Fatigue/QoL, MDS Cooperative Group. Effects of $40.000 \mathrm{UI}$ bi-weekly induction dose of epoetin alpha followed by 40.000 $\mathrm{UI}$ once weekly in low risk myelodysplastic syndrome patients. Ann Hematol 2005;84:167-76.

[28] Greenberg P, Taylor K, Larson R, et al. Phase III, randomized trial of G-CSF vs observation for myelodysplastic syndromes. Blood 1993;82:196.

[29] Willemze R, van der Lely N, Zwierzina H, Suciu S, Solbu G, $\mathrm{Gerhartz} \mathrm{H}$, et al. A randomized phase-I/II multicenter study of recombinant human granulocyte-macrophage colony-stimulating factor (GM-CSF) therapy for patients with myelodysplastic syndromes and a relatively low risk of acute leukemia. EORTC Leukemia Cooperative Group. Ann Hematol 1992;64(4): 173-80.

[30] Thomas ML. Quality of life and psychosocial adjustment in patients with myelodysplastic syndromes. Leuk Res 1998; 22(Suppl 1):S41-7.

[31] Jansen AJ, Essink-Bot ML, Beckers EA, Hop WC, Schipperus MR, Van Rhenen DJ. Quality of life measurement in patients with transfusion-dependent myelodysplastic syndromes. $\mathrm{Br} \mathrm{J}$ Haematol 2003;121(2):270-4.

[32] Worwood M. Serum ferritin. Clin Sci (Lond) 1986;70(3): 215-20.

[33] Olivieri NF, Nathan DG, MacMillan JH, et al. Survival in medically treated patients with homozygous beta-thalassemia. N Engl J Med 1994;331(9):574-8.

[34] Finelli C, Bosi C, Clissa C. Supporto trasfusionale e chelazione. In: Aloe Spiriti MA, Cortelezzi A, Finelli C, et al., editors. Sindromi mielodisplastiche. Dalla teoria alla pratica clinica. Milano: Elsevier Masson; 2008. p. 87.

[35] Cappellini MD, Cohen A, Piga A, Bejaoui M, Perrotta S, Agaoglu L, et al. A phase 3 study of deferasirox (ICL670), a once-daily oral iron chelator, in patients with beta-thalassemia. Blood 2006;107(9):3455-62.

[36] Santini V, Alessandrino PE, Angelucci E, Barosi G, Billio A, Di Maio $M$, et al. Clinical management of myelodysplastic syndromes: update of SIE, SIES, GITMO practice guidelines. Leuk Res 2010 Feb 11 [Epub ahead of print.].

[37] List A, Kurtin S, Roe DJ, Buresh A, Mahadevan D, Fuchs D, et al. Efficacy of lenalidomide in myelodysplastic syndromes. N Engl J Med 2005;352(6):549-57.

[38] Melchert $M$, Kale V, List A. The role of lenalidomide in the treatment of patients with chromosome $5 q$ deletion and other myelodysplastic syndromes. Curr Opin Hematol 2007;14(2): 123-9.

[39] Giagounidis AA, Germing U, Wainscoat JS, Boultwood J, Aul C. The 5q- syndrome. Hematology 2004;9(4):271-7.

[40] Pellagatti $A$, Jädersten $M$, Forsblom $A M$, et al. Lenalidomide inhibits the malignant clone and up-regulates the SPARC gene 
mapping to the commonly deleted region in $5 \mathrm{q}$ - syndrome patients. Proc Natl Acad Sci U S A 2007;104(27):11406-11.

[41] Ebert BL, Pretz J, Bosco J, Chang CY, Tamayo P, Galili N, et al. Identification of RPS14 as a $5 q$ - syndrome gene by RNA interference screen. Nature 2008;451(7176):335-9.

[42] Voso MT, Scardocci A, Guidi F, Zini G, Di Mario A, Pagano L, et al. Aberrant methylation of DAP-kinase in therapy-related acute myeloid leukemia and myelodysplastic syndromes. Blood 2004; 103(2):698-700.

[43] Teofili L, Martini M, Luongo M, Diverio D, Capelli G, Breccia M, et al. Hypermethylation of $\mathrm{GpG}$ islands in the promoter region of p15(INK4b) in acute promyelocytic leukemia represses p15(INK4b) expression and correlates with poor prognosis. Leukemia 2003;17(5):919-24.

[44] Kaminskas E, Farrell A, Abraham S, Baird A, Hsieh LS, Lee SL, et al., FDA. Approval summary: azacitidine for treatment of myelodysplastic syndrome subtypes. Clin Cancer Res 2005; 11(10):3604-8.

[45] Silverman LR. DNA methyltransferase inhibitors in myelodysplastic syndrome. Best Pract Res Clin Haematol 2004;17(4):585-94.

[46] Siddiqui MA, Scott LJ. Azacitidine: in myelodysplastic syndromes. Drugs 2005;65(13):1781-9.

[47] Silverman LR, Holland JF, Weinberg RS, Alter BP, Davis RB, Ellison RR, et al. Effects of treatment with 5-azacytidine on the in vivo and in vitro hematopoiesis in patients with myelodysplastic syndromes. Leukemia 1993;7(Suppl 1):21-9.

[48] Silverman LR, Demakos EP, Peterson BL, Kornblith AB, Holland $\mathrm{JC}$, Odchimar-Reissig R, et al. Randomized controlled trial of azacitidine in patients with the myelodysplastic syndrome: a study of the cancer and leukemia group B. J Clin Oncol 2002;20(10):2429-40.
[49] Fenaux P, Mufti GJ, Hellstrom-Lindberg E, Santini V, Finelli C, Giagounidis A, et al., International Vidaza High-Risk MDS Survival Study Group. Efficacy of azacitidine compared with that of conventional care regimens in the treatment of higher-risk myelodysplastic syndromes: a randomised, open-label, phase III study. Lancet Oncol 2009;10(3):223-32.

[50] Lyons RM, Cosgriff TM, Modi SS, Gersh RH, Hainsworth JD, Cohn $\mathrm{AL}$, et al. Hematologic response to three alternative dosing schedules of azacitidine in patients with myelodysplastic syndromes. J Clin Oncol 2009;27(11):1850-6.

[51] Follo MY, Finelli C, Bosi C, Martinelli G, Mongiorgi S, Baccarani $M$, et al. PI-PLCbeta-1 and activated Akt levels are linked to azacitidine responsiveness in high-risk myelodysplastic syndromes. Leukemia 2008;22(1):198-200.

[52] Follo MY, Finelli C, Mongiorgi S, Clissa C, Bosi C, Testoni N, et al. Reduction of phosphoinositide-phospholipase $\mathrm{C}$ beta1 methylation predicts the responsiveness to azacitidine in high-risk MDS. Proc Natl Acad Sci U S A 2009;106(39):16811-6.

[53] Follo MY, Finelli C, Clissa C, Mongiorgi S, Bosi C, Martinelli G, et al. Phosphoinositide-phospholipase $\mathrm{C}$ beta1 mono-allelic deletion is associated with myelodysplastic syndromes evolution into acute myeloid leukemia. J Clin Oncol 2009;27(5):782-90.

[54] Finelli C, Terenzi A, Poloni A. Trapianto di cellule staminali. In: Aloe Spiriti MA, Cortelezzi A, Finelli C, et al., editors. Sindromi mielodisplastiche. Dalla teoria alla pratica clinica. Milano: Elsevier Masson; 2008. p. 165.

[55] Cutler CS, Lee SJ, Greenberg P, Deeg HJ, Pérez WS, Anasetti C, et al. A decision analysis of allogeneic bone marrow transplantation for the myelodysplastic syndromes: delayed transplantation for low-risk myelodysplasia is associated with improved outcome. Blood 2004;104(2):579-85. 